\title{
LOCAL PARTIAL DIFFERENTIAL ALGEBRA
}

\author{
BY \\ S. HALFIN AND A. ROBINSON(1)
}

1. Introduction. An algebraic theory for the solution of initial value problems for systems of algebraic differential equations has been given in [3]. In the present paper, we extend the theory to systems of partial differential equations.

The concept of a partial differential ring with $n$ derivations has been defined by J. F. Ritt [2]. For our present purpose we have to supplement this definition by a homomorphism into a partial differential ring with $n-1$ differentiations, which corresponds to the surface on which the initial values are given. Thus, the formal setting within which we shall carry out our investigation is as follows: A localized partial differential ring (1.p.d.r.) is a system $\Omega=\left(R, R_{0}, H\right)$ such that:

$R$ is an integral domain and a partial differential ring with $n$ derivations $D_{1}, \cdots, D_{n}, n \geqq 2$;

$R_{0}$ is an integral domain and (partial, for $n \geqq 2$, ordinary, for $n=2$ ) differential ring with $n-1$ derivations, $\Delta_{1}, \cdots, \Delta_{n-1} ; H(a)$ is a homomorphic mapping of $R$ into $R_{0}, H(1)=1$;

there exists a matrix of elements of $R, \hat{A}=\left(a_{i k}\right), i=1, \cdots, n-1, k=1, \cdots, n$, such that $\left(H\left(a_{i k}\right)\right)$ is of rank $n-1$ and

$$
H\left(\sum_{k=1}^{n} a_{i k} D_{k} a\right)=\Delta_{i} H(a), \quad i=1, \cdots, n-1, \text { for all } a \in R .
$$

An 1.p.d.r. will be called normal if $a_{i k}=\delta_{i k}$, i.e.,

$$
H\left(D_{i} a\right)=\Delta_{i} H(a), \quad i=1, \cdots, n-1 .
$$

2. Equivalent systems of derivations. Let $G=\left(g_{i k}\right), i=1, \cdots, r, k=1, \cdots, n$, be a rectangular matrix whose elements belong to $R$, where $r \geqq 1$. We say that $G$ is normal with respect to $R$ if

$$
\sum_{k=1}^{n}\left(g_{j k} D_{k} g_{i l}-g_{i k} D_{k} g_{j l}\right)=0 \quad \text { for every } i, j=1, \cdots, r, \quad l=1, \cdots, n .
$$

Let $E=\left(e_{i k}\right)$ be a matrix of order $n \times n$ over $R$. We define $n$ new derivations on $R$ by

Received by the editors September 6, 1962.

(1) The research reported in this document has been sponsored in part by the Air Force Office of Scientific Research, OAR, through the European office, Aerospace Research, United States Air Force. 


$$
\hat{D}_{i}=\sum_{k=1}^{n} e_{i k} D_{k}
$$

The system $\left\langle\hat{D}_{i}\right\rangle$ is called equivalent to the system $\left\langle D_{i}\right\rangle$ if

(i) $E$ possesses an invese over $R$,

(ii) For every extension $\widetilde{R}$ of $R, \widetilde{R}$ with the derivations $\hat{D}_{1}, \cdots, \hat{D}_{n}$, is a partial differential ring.

By the definition of a partial differential ring, condition (ii) will be satisfied if and only if the $D_{i}$ commute on the elements of $\tilde{R}$.

2.4. THEOREM. The system $\left\langle\hat{D}_{t}\right\rangle$ is equivalent to the system $\left\langle D_{i}\right\rangle$ if and only if the matrix $E$ has an inverse and is normal.

Proof. We have

hence

$$
\begin{gathered}
\hat{D}_{j} \hat{D}_{i}=\sum_{k=1}^{n} e_{j k} D_{k}\left(\sum_{l=1}^{n} e_{i l} D_{l}\right)=\sum_{k=1}^{n} \sum_{l=1}^{n} e_{j k} e_{i l} D_{k} D_{l}+\sum_{l=1}^{n}\left(\sum_{k=1}^{n} e_{j k} D_{k} e_{i l}\right) D_{l}, \\
\hat{D}_{i} \hat{D}_{j}=\sum_{k=1}^{n} \sum_{l=1}^{n} e_{i k} e_{j l} D_{k} D_{l}+\sum_{l=1}^{n}\left(\sum_{k=1}^{n} e_{i k} D_{k} e_{j l}\right) D_{l}
\end{gathered}
$$

$$
\hat{D}_{j} \hat{D}_{i}-\hat{D}_{i} \hat{D}_{j}=\sum_{l=1}^{n} \sum_{k=1}^{n}\left(e_{j k} D_{k} e_{i l}-e_{i k} D_{k} e_{j l}\right) D_{l} .
$$

If $E$ is normal, the right hand side vanishes. Thus $\hat{D}_{j} \hat{D}_{i}-\hat{D}_{i} \hat{D}_{j}$ is the zero operator for every $i$ and $j$.

Conversely, if $E$ is not normal, then there exist $i_{0}, j_{0}, l_{0}$ such that

$$
\sum_{k=1}^{n}\left(e_{j_{0} k} D_{k} e_{i_{0} l_{0}}-e_{i_{0} k} D_{k} e_{j_{0} l_{0}}\right) \neq 0
$$

Let $\widetilde{R}$ be an extension of $R$ containing an element $a$ which satisffies $D_{i} a=0$ for $i \neq l_{0}$ and $D_{l_{0}} a \neq 0$. (Standard methods of partial differential algebra ensure the existence of such extensions.) Then

$$
\left(\hat{D}_{j_{0}} \hat{D}_{i_{0}}-\hat{D}_{i_{0}} \hat{D}_{j_{0}}\right) a=\sum_{k=1}^{n}\left(e_{j_{0} k} D_{k} e_{i_{0} l_{0}}-e_{i_{0} k} D_{k} e_{j_{0} l_{0}}\right) D_{l_{0}} a \neq 0
$$

and the derivations do not commute on $\tilde{R}$.

Let the system $\left\langle\hat{D}_{i}\right\rangle$ be equivalent to $\left\langle D_{i}\right\rangle$, then $R$ with the derivations $\left\langle\hat{D}_{i}\right\rangle$ forms a partial differential ring which will be denoted by $\hat{R}$.

2.6. THEOREM. If $\left\langle D_{i}\right\rangle$ is a system equivalent to $\left\langle\hat{D}_{i}\right\rangle$ and $\left\langle\tilde{D}_{i}\right\rangle$ is a system equivalent to $\left\langle\hat{D}_{i}\right\rangle$ (with respect to $\hat{R}$ ), then $\left\langle\widetilde{D}_{i}\right\rangle$ is equivalent to $\left\langle D_{i}\right\rangle$.

Proof. Let $E$ be the matrix transforming $\left\langle D_{i}\right\rangle$ to $\left\langle\hat{D}_{i}\right\rangle$ and let $F$ be the matrix transforming $\left\langle\hat{D}_{i}\right\rangle$ to $\left\langle\widetilde{D}_{i}\right\rangle ; E$ and $F$ possess inverses, hence $F E$, which trans- 
forms $\left\langle D_{i}\right\rangle$ to $\left\langle\tilde{D}_{i}\right\rangle$, possesses an inverse. If $\widetilde{R}$ is an extension of $R$, then $\tilde{R}$ regarded as a partial differential ring with the derivations $\left\langle\hat{D}_{i}\right\rangle$ is an extension of $\hat{R}$. It follows that the derivations $\left\langle\tilde{D}_{i}\right\rangle$ commute on $\tilde{R}$. This proves the theorem.

2.7. TheOrEM. If $\left\langle\hat{D}_{i}\right\rangle$ is equivalent to $\left\langle D_{i}\right\rangle$ then $\left\langle D_{i}\right\rangle$ is equivalent to $\left\langle\hat{D}_{i}\right\rangle$.

Proof. Let $E=\left(e_{i j}\right)$ be the matrix transforming $\left\langle D_{i}\right\rangle$ to $\left\langle\hat{D}_{i}\right\rangle$. By Theorem 2.5, $E$ is normal and has an inverse. Let $F=\left(f_{i j}\right)$ be the inverse matrix to $E$. $F$ transforms $\left\langle\hat{D}_{i}\right\rangle$ to $\left\langle D_{i}\right\rangle$. We shall show that $F$ is normal with respect to $\hat{R}$.

Since $E$ is normal it follows that $\hat{D}_{j} e_{i l}=\hat{D}_{i} e_{j l}$ for every $1 \leqq i, j, l \leqq n$. Let $1 \leqq r, s, t \leqq n$ be fixed, then

$$
\sum_{i=1}^{n} \sum_{j=1}^{n} \sum_{l=1}^{n} f_{r i} f_{s j} f_{l t} \hat{D}_{j} e_{i l}=\sum_{i=1}^{n} \sum_{j=1}^{n} \sum_{l=1}^{n} f_{r i} f_{s j} f_{l t} \hat{D}_{i} e_{j l} .
$$

Note that

$$
\sum_{i=1}^{n} f_{r i} \hat{D}_{j} e_{i l}+\sum_{i=1}^{n} e_{i l} \hat{D}_{j} f_{r i}=\hat{D}_{j} \sum_{i=1}^{v} f_{r i} e_{i l}=\hat{D}_{j} \delta_{r l}=0
$$

Thus,

$$
\sum_{i=1}^{n} f_{r i} \hat{D}_{j} e_{i l}=-\sum_{i=1}^{n} e_{i l} \hat{D}_{j} f_{r i}
$$

Transforming the left hand side of (2.8) we get

$$
\begin{aligned}
\sum_{i, j, k=1}^{n} f_{r i} f_{s i} f_{l t} \hat{D}_{j} e_{i l} & =\sum_{j=1}^{n} f_{s j} \sum_{l=1}^{n} f_{l t} \sum_{i=1}^{n} f_{r i} \hat{D}_{j} e_{i l}=-\sum_{j=1}^{n} f_{s j} \sum_{l=1}^{n} f_{l t} \sum_{i=1}^{n} e_{i l} \hat{D}_{j} f_{r i} \\
& =-\sum_{j=1}^{n} f_{s j} \sum_{i=1}^{n} \hat{D}_{j} f_{r i} \delta_{i t}=-\sum_{j=1}^{n} f_{s j} \hat{D}_{j} f_{r t} .
\end{aligned}
$$

In the same manner, the right hand side equals $-\sum_{j=1}^{n} f_{r j} \hat{D}_{j} f_{s t}$. Hence $\sum_{j=1}^{n} f_{s j} \hat{D}_{j} f_{r t}=\sum_{j=1}^{n} f_{r j} \hat{D}_{j} f_{s t}$, proving that $F$ is normal with respect to $\hat{R}$. This shows that the system $\left\langle D_{i}\right\rangle$ is equivalent to $\left\langle\hat{D}_{i}\right\rangle$.

3. Normalization. Let $\Omega=\left(R, R_{0}, H\right)$ and $\Omega^{\prime}=\left(R^{\prime}, R_{0}^{\prime}, H^{\prime}\right)$ be two l.p.d.r. $\Omega^{\prime}$ is called an extension of $\Omega$ if $R^{\prime}$ and $R_{0}^{\prime}$ are extensions of $R$ and $R_{0}$, respectively, $H^{\prime}(a)=H(a)$ for every $a \in R$, and if condition (1.1) is valid in $\Omega$ for the same matrix $\hat{A}$.

In this section it will be shown that if the matrix $A$ is normal, then $\Omega$ can be extended to an l.p.d.r. $\Omega^{*}=\left(R^{*}, R_{0}^{*}, H^{*}\right)$ which is capable of normalization in the following sense: There exists in $R^{*}$ a system of derivations $\bar{D}_{1}, \cdots, \bar{D}_{n}$, equivalent to $D_{1}, \cdots, D_{n}$, such that if $\bar{R}^{*}$ is the partial differential ring consisting of the ring $R^{*}$ with the derivations $\bar{D}_{1}, \cdots, \bar{D}_{n}$, then $\bar{\Omega}^{*}=\left(\bar{R}^{*}, R^{*}, H^{*}\right)$ is a normal 1.p.d.r. We prove two lemmas which will be useful later: 
3.1. Lemma. Let $R$ be a ring generated by a set of elements $A$ in the sense that every element of $R$ can be expressed as a rational combination of elements of $A$. Let $D_{1}$ and $D_{2}$ be two derivations on $R$ whose range is in some extension of $R$, commuting on the elements of $A$.

Then $D_{1}$ and $D_{2}$ commute on $R$.

Proof. If $D_{1}$ and $D_{2}$ commute on $a$ and $b$, then they commute on $a \pm b$, $a b$ and on $a^{-1}$ (if it exists in $R$ ). Therefore they commute on every rational combination of elements of $A$, which belong to $R$.

3.2. LeMma. Let $R$ be a partial differential ring with $n$ derivations $D_{1}, \cdots, D_{n}$, and let $R$ be generated by the set $A$ in the sense of Lemma 3.1. Let $R_{0}$ be a partial differential ring with $n-1$ derivations $\Delta_{1}, \cdots, \Delta_{n-1}$. Let $H$ be a homomorphism of $R$ into $R_{0}$, and let condition (1.1) be a satisfied_by all elements $a$ of $A$. Then condition (1.1) is satisfied by all the elements of $R$.

Proof. It is easily seen that if condition (1.1) is satisfied by $a$ and $b$, then it is satisfied by $a \pm b, a b$ and $a^{-1}$ (if $a$ has an inverse in $R$ ). Therefore condition (1.1) is satisfied by all elements of $R$.

3.3. THEOREM. Let $R$ be an integral domain and a partial differential ring with the derivations $D_{1}, \cdots, D_{n}$. Let $\hat{A}=\left(a_{i k}\right), i=1, \cdots, n-1, k=1, \cdots, n$, be $a$ matrix normal over $R$ of rank $n-1$. Then there exist elements $a_{n 1}, \cdots, a_{n n}$ in some differential extension $R^{*}$ of $R$, such that the matrix $A=\left(a_{i k}\right), i=1, \cdots, n$, $k=1, \cdots, n$, is normal and has an inverse in $R^{*}$.

Proof. Let $\left\langle x_{i j}\right\rangle, i=1, \cdots, n, j=1,2, \cdots$, be an infinite set of elements transcendental over $R$. Let $M$ be the matrix

$$
\left[\begin{array}{cc}
a_{11}, \cdots, a_{1 n} \\
\vdots & \\
a_{n-11}, \cdots, a_{n-1 n} \\
& x_{11}, \cdots, x_{n 1}
\end{array}\right]
$$

over $R\left[x_{11}, \cdots, x_{n 1}\right]$. Let $d=|M|$, then $d \neq 0$, and since $R\left[x_{11}, \cdots, x_{n 1}\right]$ is an integral domain, $d^{-1}$ can be adjoined to it.

Let $\left(R_{i}\right)$ be an increased sequence of rings defined in the following manner: $R_{0}=R, R_{1}=R\left[x_{11}, \cdots, x_{n 1}\right]\left[d^{-1}\right]$, and for $i>1, R_{i}=R_{i-1}\left[x_{1 i}, \cdots, x_{n i}\right]$.

We introduce a new derivation from $R_{0}$ into $R_{1}$ :

$$
\delta(a)=\sum_{u=1}^{n} x_{u 1} D_{u} a, \quad a \in R,
$$

and we define the derivations $D_{1}, \cdots, D_{n}$ on the elements $x_{11}, \cdots, x_{n 1}$ by requiring that for every fixed $l, l=1, \cdots, n$, the following system of $n$ linear equations will be satisfied: 


$$
\begin{array}{ll}
\sum_{k=1}^{n} a_{i k} D_{k} x_{l 1}=\delta a_{i l}, & i=1, \cdots, n-1, \\
\sum_{k=1}^{n} x_{k 1} D_{k} x_{l 1} & =x_{l 2} .
\end{array}
$$

The matrix of the system is $M$, and since $|M|^{-1} \in R_{1}$ the system possesses a unique solution in $R_{2}$. Using this solution we extend the definition of the derivations $D_{1}, \cdots, D_{n}$ on $R_{1}$, with range in $R_{2} . \delta$ is extended to a derivation of $R_{1}$ into $R_{2}$ by Definition (3.4).

Suppose that the derivations $D_{1}, \cdots, D_{n}$ have been extended to $R_{t-1}(t \geqq 1)$ so that every $D_{i}$ transforms $R_{k}$ into $R_{k+1}(k=0, \cdots, t-1) . \delta$ can now be defined on $R_{t-1}$ by Definition (3.4). $\delta^{t}$ is then an operator transforming $R$ into $R_{t}$.

We define the derivations $D_{1}, \cdots, D_{n}$ on $x_{1 t}, \cdots, x_{n t}$ so that the following system of linear equations is satisfied:

$$
\begin{array}{ll}
\sum_{k=1}^{n} a_{i k} D_{k} x_{l t}=\delta^{t} a_{i l}, & i=1, \cdots, n-1, \\
\sum_{k=1}^{n} x_{k 1} D_{k} x_{l t}=x_{l t+1} . &
\end{array}
$$

For every fixed $l$, this is a system of $n$ equations for $D_{k} x_{l t}$, with coefficients in $R_{t+1}$. The matrix of the system is $M$; therefore, there exists a unique solution for the system in $R_{t+1}$. Accordingly, the definition of $D_{1}, \cdots, D_{n}$ and $\delta$ can be extended to $R_{t}$, and their range will be in $R_{t+1}$.

Let $R^{*}=\bigcup_{i=0}^{\infty} R_{i}$. Having extended $D_{1}, \cdots, D_{n}$ to $R^{*}$ by induction, we are going to show that the derivations commute on $R^{*}$.

By Lemma 3.1, it is sufficient to show that the derivations commute on the $x_{i k}$, and this will be proved by induction. Suppose that the derivations commute on every $x_{i k}$ for $k<t(t \geqq 1)$. Then by Lemma 3.1, they commute on $R_{t-1}$. We shall prove that they commute on $x_{1 t}, \cdots, x_{n t}$.

We first prove that

$$
\sum_{k=1}^{n} a_{j k} D_{k} \delta^{t} a_{i l}=\sum_{k=1}^{n} a_{i k} D_{k} \delta^{t} a_{j l}, \quad i, j=1, \cdots, n-1, \quad l=1, \cdots, n .
$$

Indeed

$$
\begin{aligned}
\sum_{k=1}^{n} a_{j k} D_{k} \delta^{t} a_{i l} & =\sum_{k=1}^{n} a_{j k} D_{k} \sum_{u=1}^{n} x_{u 1} D_{u} \delta^{t-1} a_{i l} \\
& =\sum_{k=1}^{n} \sum_{u=1}^{n} a_{j k}\left(D_{k} x_{u 1}\right) D_{u} \delta^{t-1} a_{i l}+\sum_{k=1}^{n} \sum_{u=1}^{n} a_{j k} x_{u 1} D_{k} D_{u} \delta^{t-1} a_{i} \\
& =\sum_{u=1}^{n}\left(\delta a_{j}{ }^{t-1} a_{i l}+\sum_{k=1}^{n} a_{j k} \delta D_{k} \delta^{t-1} a_{i l}=\delta\left(\sum_{k=1}^{n} a_{j k} D_{k} \delta^{t-1} a_{i l}\right)\right.
\end{aligned}
$$

( $\delta^{t-1} a_{i l} \in R_{t-1}$ and so the derivations commute on it). 
Continuing in this way we get

and similarly

$$
\sum_{k=1}^{n} a_{j k} D_{k} \delta^{t} a_{i l}=\delta^{t} \sum_{k=1}^{n} a_{j k} D_{k} a_{i l},
$$

$$
\sum_{k=1}^{n} a_{i k} D_{k} \delta^{t} a_{j l}=\delta^{t} \sum_{k=1}^{n} a_{i k} D_{k} a_{j l}
$$

The right hand sides are equal since $\hat{A}$ is normal. This proves (3.7).

Using equations (3.6) we get

$$
\begin{aligned}
\sum_{k=1}^{n} a_{j k} D_{k} \delta^{t} a_{i l} & =\sum_{k=1}^{n} a_{j k} D_{k} \sum_{u=1}^{n} a_{i u} D_{u} x_{l t} \\
& =\sum_{k=1}^{n} \sum_{u=1}^{n} a_{j k}\left(D_{k} a_{i u}\right) D_{u} x_{l t}+\sum_{k=1}^{n} \sum_{u=1}^{n} a_{j k} a_{i u} D_{k} D_{u} x_{l t}
\end{aligned}
$$

The right hand side of (3.7) yields a similar expression. Hence

$$
\begin{aligned}
\sum_{k=1}^{n} \sum_{u=1}^{n} a_{j k}\left(D_{k} a_{i u}\right) D_{u} x_{l t} & +\sum_{k=1}^{n} \sum_{u=1}^{n} a_{j k} a_{i u} D_{k} D_{u} x_{l t} \\
= & \sum_{k=1}^{n} \sum_{u=1}^{n} a_{i k}\left(D_{k} a_{j u}\right) D_{u} x_{l t}+\sum_{k=1}^{n} \sum_{u=1}^{n} a_{i k} a_{j u} D_{k} D_{u} x_{l t}
\end{aligned}
$$

The first sum on the right hand side is equal to the first sum on the left hand side since $\hat{A}$ is normal. Therefore

$$
\sum_{u=1}^{n} \sum_{k=1}^{n} a_{i k} a_{j u}\left(D_{k} D_{u}-D_{u} D_{k}\right) x_{i t}=0, i, j=1, \cdots, n-1, l=1, \cdots, n .
$$

Now let us calculate the following expressions:

$$
\begin{aligned}
& \sum_{u=1}^{n} a_{j u} D_{u} \sum_{k=1}^{n} x_{k 1} D_{k} x_{l t}=\sum_{u=1}^{n} a_{j u} D_{u} x_{l t+1}=\delta^{t+1} a_{j l}, \\
& \sum_{u=1}^{n} x_{u 1} D_{u} \sum_{k=1}^{n} a_{j k} D_{k} x_{l t}=\sum_{u=1}^{n} x_{u 1} D_{u} \delta^{t} a_{j l}=\delta^{t+1} a_{j l} .
\end{aligned}
$$

Thus the left hand sides are equal, and by expanding them we get

$$
\begin{aligned}
\sum_{u=1}^{n} \sum_{k=1}^{n} a_{j u}\left(D_{u} x_{k 1}\right) D_{k} x_{l t} & +\sum_{u=1}^{n} \sum_{k=1}^{n} a_{j u} x_{k 1} D_{u} D_{k} x_{l t} \\
= & \sum_{u=1}^{n} \sum_{k=1}^{n} x_{u 1}\left(D_{u} a_{j k}\right) D_{k} x_{l t}+\sum_{u=1}^{n} \sum_{k=1}^{n} x_{u 1} a_{j k} D_{u} D_{k} x_{l t} .
\end{aligned}
$$

The first term on the right hand side is equal to the first term on the left hand side since both are equal to $\sum_{k=1}^{n} \delta\left(a_{j k}\right) D_{k} x_{l t}$. Thus 


$$
\sum_{u=1}^{n} \sum_{k=1}^{n} x_{k 1} a_{j u}\left(D_{k} D_{u}-D_{u} D_{k}\right) x_{l t}=0, \quad j=1, \cdots, n-1, \quad l=1, \cdots, n .
$$

For a fixed $l$, let $B$ be the $n \times n$ matrix whose $(k, u)$ element is $\left(D_{k} D_{u}-D_{u} D_{k}\right) x_{l t}$. Then equalities (3.8) and (3.9) state that $M B M^{*}=0$ ( $M^{*}$ is the transposed matrix). Since $M$ is regular, $B=0$. This proves that the derivations commute on $x_{1 t}, \cdots, x_{n t}$.

Thus $R^{*}$ is a differential extension of $R$. Putting $a_{n k}=x_{k 1}, k=1, \cdots, n$, the matrix $A=\left(a_{i k}\right), 1 \leqq i, k \leqq n$, is equal to the matrix $M$ and thus has an inverse in $R^{*}$. Moreover, $A$ is normal. Conditions (2.1) are satisfied for $1 \leqq i, j \leqq n-1$ because $\hat{A}$ is normal, and for $i=n, j \leqq n-1$ and $j=n, i \leqq n-1$ because

$$
\sum_{k=1}^{n} a_{j k} D_{k} x_{l 1}=\delta a_{j l}=\sum_{k=1}^{n} x_{k 1} D_{k} a_{j l} .
$$

This completes the proof of Theorem 3.3.

We now state and prove the fundamental theorem.

3.10. TheOREM. Let $\Omega=\left(R, R_{0}, H\right)$ be an l.p.d.r. such that its transforming matrix $\hat{A}$ is normal. Then there exists an extension $\Omega^{*}=\left(R^{*}, R_{0}^{*}, H^{*}\right)$ for $\Omega$ such that $\Omega^{*}$ can be normalized.

Proof. $R$ and the matrix $\hat{A}$ satisfy the conditions of Theorem 3.3. We define $R^{*}$ as in the proof of that theorem, using the same notation. Let $\left(v_{i j}\right), i=1, \cdots, n$, $j=1,2, \cdots$, be a set of elements transcendental over $R_{0}$. Let

$$
d_{0}=\left|\begin{array}{l}
H\left(a_{11}\right) \cdots H\left(a_{1 n}\right) \\
\cdot \\
\cdot \\
H\left(a_{n-11}\right) \cdots H\left(a_{n-1 n}\right) \\
v_{11 \ldots v_{n 1}}
\end{array}\right| .
$$

Then $d_{0}$ belongs to $R_{0}\left[v_{i j}\right]$. The matrix $H(\hat{A})$ is of rank $n-1$, thus $d_{0} \neq 0$, and since $R_{0}\left[v_{i j}\right]$ is an integral domain, we can adjoin $d_{0}^{-1}$ to it.

We define $R_{0}^{*}=R_{0}\left[v_{i j}\right]\left(d_{0}^{-1}\right)$.

Let $H^{*}$ be a homomorphism of $R^{*}$ into $R_{0}^{*}$ defined in the following way: $H^{*}(a)=H(a)$ for $a \in R, H^{*}\left(x_{i j}\right)=v_{i j}$ for $i=1, \cdots, n, j=1,2, \cdots$. This defines $H^{*}$ on $R\left[x_{i j}\right]$. Moreover, we have $H^{*}(d)=d_{0}$. Accordingly, $H^{*}$ can be defined on $d$ $H^{*}\left(d^{-1}\right)=d_{0}^{-1}$, and thus $H^{*}$ can be extended to a homomorphism of $R^{*}$ into $R_{0}^{*}$.

We extend $\Delta_{1}, \cdots, \Delta_{n-1}$ to derivations on $R_{0}^{*}$ by defining

$$
\Delta_{i} v_{l t}=H^{*}\left(\delta^{t} a_{i l}\right), \quad i=1, \cdots, n-1, l=1, \cdots, n, t=1,2, \cdots .
$$

We are going to prove that (1.1) is satisfied for every $a \in R^{*}$. Since (1.1) is satisfied by every $a \in R$, it is sufficient to show that it is valid for every $x_{l t}$ (Lemma 
3.2). Indeed, $H^{*}\left(\sum_{k=1}^{n} a_{i k} D_{k} x_{l t}\right)=H^{*}\left(\delta^{t} a_{i l}\right)=\Delta_{i} v_{l t}=\Delta_{i}\left(H^{*}\left(x_{l t}\right)\right)$. Finally we shall show that the derivations $\Delta_{1}, \cdots, \Delta_{n-1}$ commute on $R_{0}^{*}$. By Lemma 3.1 , it is sufficient to prove that they commute on every $v_{l t}$ :

$$
\begin{aligned}
& \Delta_{j} \Delta_{i} v_{l t}=\Delta_{j} H^{*}\left(\delta^{t} a_{i l}\right)=H^{*}\left(\sum_{k=1}^{n} a_{j k} D_{k} \delta^{t} a_{i l}\right), \\
& \Delta_{i} \Delta_{j} v_{l t}=H^{*}\left(\sum_{k=1}^{n} a_{i k} D_{k} \delta^{t} a_{j l}\right) .
\end{aligned}
$$

The right hand sides are equal (by (3.7)); hence $\left(\Delta_{i} \Delta_{j}-\Delta_{j} \Delta_{i}\right) v_{l t}=0$. Thus $R_{0}^{*}$ is a differential extension of $R_{0}$ and therefore $\Omega^{*}=\left(R^{*}, R_{0}^{*}, H^{*}\right)$ is an extension of $\Omega$. The matrix $A$ as defined in the proof of 3.3 is normal and possesses an inverse in $R^{*}$; hence the system of derivations

$$
\bar{D}_{i}=\sum_{j=1}^{n} a_{i j} D_{j}, \quad i=1, \cdots, n,
$$

is equivalent to the system $D_{1}, \cdots, D_{n}$. If we define $\bar{R}^{*}$ as the ring $R^{*}$ with the derivations $\bar{D}_{1}, \cdots, \bar{D}_{n}$, then $\left(\bar{R}^{*}, R_{0}^{*}, H^{*}\right)$ is a normal l.p.d.r., since

$$
H^{*}\left(\bar{D}_{i}(a)\right)=H^{*}\left(\sum_{j=1}^{n} a_{i j} D_{j} a\right)=\Delta_{i} H^{*}(a) \text { for every } a \in \bar{R}^{*} .
$$

This proves Theorem (3.10).

The vector $\left(a_{n 1}, \cdots, a_{n n}\right)$ is called a transversal vector, the derivations $\bar{D}_{1}, \cdots, \bar{D}_{n-1}$ will be called inner derivations, and $\bar{D}_{n}$ a transversal derivation.

From now on we shall treat only localized partial differential rings which admit normalization.

4. Polynomial extensions. The ring of differential polynomials in $m$ variables $u_{1}, \cdots, u_{m}$ over a partial differential ring was defined in [2] and will be denoted by $R\left\{u_{1}, \cdots, u_{m}\right\}$.

Let $\hat{D}_{1}, \cdots, \hat{D}_{n}$ by a system of derivations equivalent to $D_{1}, \cdots, D_{n}$ over $R$ and let $\hat{R}$ be the differential ring consisting of the ring $R$ with the derivations $\hat{D}_{1}, \cdots, \hat{D}_{n}$. Then we can construct the two polynomial extensions,

$$
\begin{array}{lrl}
R & \left.u_{1}, \cdots, u_{m}\right\}=R\left[D^{\left(\alpha_{1}, \ldots, \alpha_{n}\right)} u_{i}\right] & i=1, \cdots, n, \\
\hat{R}\left\{y_{1}, \cdots, y_{m}\right\}=\hat{R}\left[\hat{D}^{\left(\alpha_{1}, \ldots, \alpha_{n}\right)} y_{i}\right] & \alpha_{j}=0,1, \cdots,
\end{array}
$$

$D^{\left(\alpha_{1}, \ldots, \alpha_{n}\right)}$ being the operator $D_{1}^{\alpha_{1}} \cdots D_{n}^{\alpha_{n}}$, and similarly $\hat{D}^{\left(\alpha_{1}, \ldots, \alpha_{n}\right)}$. Each of these rings is a partial differential ring with each of the two systems of derivations. This follows from the definition of equivalence between systems, taking into account Theorem 2.7.

We prove 
4.1. THEOREM. $R\left\{u_{1}, \cdots, u_{m}\right\}$ and $\hat{R}\left\{y_{1}, \cdots, y_{m}\right\}$ are differentially isomorphic with regard to each of the two systems of derivations.

Proof. We define

$$
\begin{aligned}
& \phi: R\left\{u_{1}, \cdots, u_{m}\right\} \longrightarrow \hat{R}\left\{y_{1}, \cdots, y_{m}\right\}, \\
& \psi: \hat{R}\left\{y_{1}, \cdots, y_{m}\right\} \longrightarrow R\left\{u_{1}, \cdots, u_{m}\right\},
\end{aligned}
$$

in the following way: $\phi(a)=\psi(a)=a$ for the elements of $R$ (which are also the elements of $\hat{R}$ ) and

$$
\begin{aligned}
& \phi\left(D^{\left(\alpha_{1}, \ldots, \alpha_{n}\right)} u_{i}\right)=D^{\left(\alpha_{1}, \ldots, \alpha_{n}\right)} y_{i}, \\
& \psi\left(\hat{D}^{\left(\alpha_{1}, \ldots, \alpha_{n}\right)} y_{i}\right)=\hat{D}^{\left(\alpha_{1}, \ldots, \alpha_{n}\right)} u_{i} .
\end{aligned}
$$

With these definitions $\phi$ can be extended to a homomorphism of $R\left\{u_{1}, \cdots, u_{m}\right\}$ into $\hat{R}\left\{y_{1}, \cdots, y_{m}\right\}$, and $\psi$ can be extended to a homomorphism in the other direction.

We shall show that $\phi$ commutes with the derivations $D_{1}, \cdots, D_{n}$. For $a \in R$, $\phi\left(D_{j} a\right)=D_{j}(a)=D_{j} \phi(a)$, and

$$
\begin{aligned}
\phi\left(D_{j} D^{\left(\alpha_{1}, \ldots, \alpha_{n}\right)} u_{i}\right) & =\phi\left(D^{\left(\alpha_{1}, \ldots, \alpha_{j}+1, \ldots, \alpha_{n}\right)} u_{i}\right)=D^{\left(\alpha_{1}, \ldots, \alpha_{j}+1, \ldots, \alpha_{n}\right)} y_{i} \\
& =D_{j}\left(\phi D^{\left(\alpha_{1}, \ldots, \alpha_{n}\right)} u_{i}\right) .
\end{aligned}
$$

Moreover, if $\phi$ and $D_{j}$ commute on $p$ and $q$, it is easy to see that they commute on $p+q$ and $p \cdot q$. Therefore they commute on every element of $R\left\{u_{1}, \cdots, u_{m}\right\}$. $\phi$ also commutes with $\hat{D}_{1}, \cdots, \hat{D}_{n}$ since

$$
\phi \hat{D}_{i}=\sum_{j=1}^{n} \phi\left(b_{i j}\right) \phi D_{j}=\sum_{j=1}^{n} b_{i j} D_{j} \phi=\hat{D}_{i} \phi .
$$

In the same way it can be shown that $\psi$ commutes with the $\left\langle D_{i}\right\rangle$ and the $\left\langle\hat{D}_{i}\right\rangle$. $\psi \phi$ is the identity on $R\left\{u_{1}, \cdots, u_{m}\right\}$, because $\psi \phi(a)=a$ for $a \in R$ and $\psi \phi\left(D^{\left(\alpha_{1}, \ldots, \alpha_{n}\right)} u_{i}\right)=D^{\left(\alpha_{1}, \ldots, \alpha_{n}\right)}\left(\psi \phi u_{i}\right)=D^{\left(\alpha_{1}, \ldots, \alpha_{n}\right)} u_{i}$. Similarly $\phi \psi$ is the identity on $\hat{R}\left\{y_{1}, \cdots, y_{m}\right\}$. Therefore $\phi$ is a one-to-one correspondence "on". This completes the proof.

We are now in a position to define the polynomial extension of an 1.p.d.r. $\Omega$. We first introduce the polynomial extension for a particular normalization $\bar{\Omega}=\left(\bar{R}, R_{0}, H\right)$ of $\Omega$ :

$$
\begin{array}{r}
\bar{\Omega}^{\prime}=\bar{\Omega}\{y, z\}=\left(\bar{R}^{\prime}, R_{0}^{\prime}, \bar{H}^{\prime}\right), \quad \text { where } \bar{R}^{\prime}=\bar{R}\left\{y_{1}, \cdots, y_{m}\right\}, \quad \bar{R}_{0}^{\prime}=R_{0}\left\{z_{i k}\right\}, \\
i=1, \cdots, m, \quad k=0,1,2, \cdots,
\end{array}
$$

and $\bar{H}^{\prime}$ is the homomorphism defined by $\bar{H}^{\prime}(a)=H(a)$ for $a \in R$ and

$$
\bar{H}^{\prime}\left(D^{\left(\alpha_{1}, \ldots, \alpha_{n}\right)} y_{i}\right)=\Delta^{\left(\alpha_{1}, \ldots, \alpha_{n}-1\right)} z_{i \alpha .} .
$$


It is easy to see that $\bar{\Omega}^{\prime}$ is a normal l.p.d.r. extending $\bar{\Omega}$.

The polynomial ring of $\Omega$ is now defined by $\Omega^{\prime}=\Omega\{u, z\}=\left(R^{\prime}, R_{0}^{\prime}, H^{\prime}\right)$, where $R^{\prime}=R\left\{u_{1}, \cdots, u_{m}\right\}, R_{0}^{\prime}$ is the same as before, and $H^{\prime}=\bar{H}^{\prime} \phi, \phi$ being the differential isomorphism from $R\left\{u_{1}, \cdots, u_{m}\right\}$ to $\bar{R}\left\{y_{1}, \cdots, y_{m}\right\}$ as defined in the proof of Theorem 4.1. $\Omega^{\prime}$ is an extension of $\Omega$ since $R^{\prime} \supseteq R, R_{0} \supseteq R_{0}, H^{\prime}(a)=H(a)$, for the elements of $R$, and for every $p \in R^{\prime}$,

$$
H^{\prime}\left(\sum_{k=1}^{n} a_{i k} D_{k} p\right)=\bar{H}^{\prime} \phi\left(\bar{D}_{i} p\right)=\bar{H}^{\prime} \widetilde{D}_{i} \phi(p)=\Delta_{i} \bar{H}^{\prime} \phi(p)=\Delta_{i} H^{\prime}(p)
$$

This shows that condition (1.1) is satisfied.

5. $S$-ideals. Let $R$ be a partial differential ring with unit element and let $S$ be a nonempty multiplicative subset of $R$ which does not include 0 .

Given any set $K \subseteq R$, we define $K_{S}$ as the set of all $b \in R$ such that $a b \in K$ for some $a \in S$. An ideal $J$ in $R$ will be called an $S$-ideal if $J_{S} \subseteq J$. A perfect (prime) ideal which is an $S$-ideal is called $S$-perfect (prime).

We have as in $[3, \S 3]$ :

5.1. If $J$ is an ideal, then $J_{S}$ is an $S$-ideal which includes $J$.

5.2. If $J$ is a perfect ideal, then $J_{S}$ is $S$-perfect.

Next we have

5.3. A prime ideal $J \neq R$ is an $S$-ideal if and only if $J \cap S$ is empty.

Indeed if $a=a \cdot 1 \in J \cap S$, then $1 \in J, J=R$ (this is true for all $S$-ideals). Conversely, if $J \cap S$ is empty, then $a b \in J, a \in S$ implies $a \notin J, b \in J$, and so $J$ is an $S$-ideal.

5.4. THEOREM. Every $S$-perfect ideal $J$ is the intersection of $S$-prime ideals which are minimal over $J$ (as prime ideals).

Proof. It is known that every perfect ideal $J$ is the intersection of prime ideals. (See [2], where the theorem is proved for ordinary differential rings. The proof is similar for partial differential rings.) A familiar argument then shows that we may restrict the set of prime ideals to those prime ideals which are minimal over $J$. We are going to show that in case $J$ is $S$-perfect, these prime ideals are also $S$-ideals.

Indeed, let $J^{\prime}$ be a prime-ideal which is minimal over $J$. If $J=R$, then $J^{\prime}=J$ is an $S$-ideal, trivially. Suppose $J \neq R$, so that $J^{\prime} \neq R$, and let $T=R-J^{\prime}$. $T$ is multiplicative since $J^{\prime}$ is prime. Let $T^{\prime}$ be the set of all products $a b$ where $a \in S, b \in T$, and let $T_{1}=T^{\prime} \cup T$. Then $T_{1}$ is the multiplicative set which is generated by $S$ and $T . T_{1} \cap J=\left(T^{\prime} \cap J\right) \cup(T \cap J)$ is empty for $T \cap J$ is empty by the definition of $T$, and $c \in T^{\prime} \cap J, c=a b, a \in S, b \in T$ implies $b \in J$, which is impossible. By a familiar argument, there exists a prime ideal $J^{*}$ which includes $J$ and excludes $T_{1}$. But then $J^{\prime} \supseteq J^{*}$ and so $J^{\prime}=J^{*}$ since $J^{\prime}$ is minimal over $J$. We conclude that $J^{\prime} \cap S \subseteq J^{\prime} \cap T_{1}$ is empty. Hence $J^{\prime}$ is $S$-ideal, by 5.3. 
Alternatively, we may prove 5.4 by the methods of $[3, \S 3]$, without the bracketed qualification as prime ideals. This yields a slightly weaker result.

6. Theory of bi-ideals. Let $\Omega=\left(R, R_{0}, H\right)$ be any given 1.p.d.r. and let $S$ be a multiplicative subset of $R, 0 \notin S$. A bi-ideal is defined similarly as in [3, §4]. Thus, $\left(J, J_{0}\right)$ is a bi-ideal if $J$ is an $S$-perfect ideal in $R$ and $J_{0}$ is a perfect ideal in $R_{0}$ such that $H(J) \subseteq J_{0}$. We introduce a partial ordering in the set of bi-ideals as in [3] using the same notation.

Let $K \subseteq R, K_{0} \subseteq R_{0}$. Then the bi-ideal $\alpha$ which is generated by $K$ and $K_{0}$ is given by

$$
\alpha=\left(\{K\}_{S},\left\{H\left(\{K\}_{S}\right) \cup K_{0}\right\}\right) .
$$

Prime and maximal bi-ideals are defined as in [3]. We have the theorem

\subsection{Every proper bi-ideal $\alpha$ is included in a maximal proper bi-ideal.}

Next we prove

6.3. THEOREM. Let $T_{0}$ be a nonempty multiplicative subset of $R_{0}$ and let the bi-ideal $\alpha=\left(J, J_{0}\right)$ be maximal with respect to the exclusion of $T_{0}$ from $J_{0}$. Then $\alpha$ is prime.

Proof. Since $J_{0}$ excludes $T_{0}$ there exists a prime ideal $J_{0}^{\prime}$ in $R_{0}$ such that $J_{0}^{\prime} \supseteq J_{0}$ and $J_{0}^{\prime} \cap T_{0}=\varnothing$. Let $T=H^{-1}\left(R_{0}-J_{0}^{\prime}\right)$, then $T$ is multiplicative. Indeed, if $H\left(t_{1}\right) \in R_{0}-J_{0}^{\prime}, H\left(t_{2}\right) \in R_{0}-J_{0}^{\prime}$, then $H\left(t_{1} t_{2}\right)=H\left(t_{1}\right) H\left(t_{2}\right) \in R_{0}-J_{0}$ since $J_{0}^{\prime}$ is prime. $J$ excludes $T$ for if $a \in J$, then $H(a) \in J_{0}$, $\subseteq J_{0}^{\prime}$, and so $a \notin T$.

Let $J^{\prime}$ be any prime ideal which excludes $T$ and is minimal over $J$. (Such $J^{\prime}$ exists by standard ideal theory.) Since $J$ is $S$-perfect it follows from the proof of 5.4 above that $J^{\prime}$ is $S$-prime. $H\left(J^{\prime}\right)$ excludes $R_{0}-J_{0}^{\prime}$ since $J^{\prime}$ excludes $T$. Hence $H\left(J^{\prime}\right) \subseteq J_{0}^{\prime}$ and $\alpha^{\prime}=\left(J^{\prime}, J_{0}^{\prime}\right)$ is a prime bi-ideal. But $\alpha<\alpha^{\prime}$ and $J_{0}^{\prime}$ excludes $T_{0}$. Hence, $\alpha^{\prime}=\alpha$ in view of the maximal property of $\alpha$.

For $T_{0}=1$ we obtain from 6.3 as a special case:

6.4. Every maximal proper bi-ideal is prime.

Combining 6.2 and 6.4 we obtain:

6.5. Every proper bi-ideal is included in a prime (proper) bi-ideal.

The closure $\bar{\alpha}$ of a bi-ideal $\alpha$ is defined as in [3]. Theorem 4.5 and 4.6 of that paper are valid in our present theory, and for the same reasons.

7. Polynomial bi-ideals. Let $\Omega=\left(R, R_{0}, H\right)$ be any 1.p.d.r. Throughout this section, we shall suppose that $R_{0}$ is a field. If this is not the case from the outset we can clearly achieve it by passing to the field of quotients.

Let $\Omega^{\prime}=\Omega\{u, z\}=\left(R^{\prime}, R_{0}^{\prime}, H^{\prime}\right)$ be the polynomial extension of $\Omega$ which is obtained by the adjunction of the $m$ differential indeterminates $u_{1}, \cdots, u_{m}$, and let 
$S=R-\langle 0\rangle$. Let $R^{*}$ be the field of quotients of $R$, and let $R^{\prime}$ and $R^{* \prime}$ be the differential rings $R\{u\}$ and $R^{*}\{u\}$, respectively. Then the relation between the ideals of $R^{\prime}$ and of $R^{* \prime}$ is described by the following theorem:

7.1. THEOREM. $J$ is an $S$-ideal in $R^{\prime}$ if and only if it is of the form $J=J^{*} \cap R^{\prime}$ where $J^{*}$ is an ideal in $R^{*} . J$ is perfect (prime) if and only if $J^{*}$ is perfect (prime). The correspondence $J^{*} \rightarrow J$ is one-to-one.

Proof. Let $J^{*}$ be any ideal in $\mathrm{R}^{* \prime}$ and let $J=J^{*} \cap R^{\prime}$. We see without difficulty that $J$ is an ideal and that if $J^{*}$ is differential (or perfect, or prime), then $J$ is differential (or perfect, or prime). Now let $a b \in J$ where $a \in S$ and $b \in R^{\prime}$. Then $a b \in J^{*}, a^{-1} \in R^{*}$ and so $b=a^{-1}(a b) \in J^{*}, b \in J^{*} \cap R^{\prime}=J$. This shows that $J$ is an $S$-ideal.

Conversely, let $J$ be an $S$-ideal in $R^{\prime}$ and let $J^{*}$ be the set of all products $a^{-1} b$ where $a \in R-\langle 0\rangle=S$ and $b \in J$. It will be seen that $J^{*}$ is an ideal in $R^{* \prime}$. Moreover, if $a^{-1} b \in R^{\prime}$, then $a\left(a^{-1} b\right) \in J$ and so $a^{-1} b \in J, J \supseteq J^{*} \cap R^{\prime}$. Since $J \subseteq J^{*}$, we conclude that $J=J^{*} \cap R^{\prime}$. The correspondence between the ideals $J$, $J^{*}$ established in this way is one-to-one for if $J_{1}^{*}, J_{2}^{*}$ determine the same $J$, let $c \in J_{1}^{*}, c=a^{-1} b$, where $a \in S, b \in R^{\prime}$. Then $b \in J_{1}^{*}$ and so $b \in J_{1}^{*} \cap R^{\prime}=J_{2}^{*} \cap R^{\prime} \subseteq J_{2}^{*}$. Hence $c=a^{-1} b \in J_{2}^{*}, J_{1}^{*} \subseteq J_{2}^{*}$, and similarly $J_{2}^{*} \subseteq J_{1}^{*}$, so we have $J_{2}^{*}=J_{1}^{*}$. Also, whenever $J$ is differential (perfect, prime) $J^{*}$ is differential (perfect, prime). This completes the proof of 7.1.

7.2. THEOREM. If $R^{* \prime}$ satisfies Ithe finite ascending chain condition for perfect ideals, then $R^{\prime}$ satisfies the finite ascending chain condition for $S$-perfect ideals.

Proof. Let $J_{1} \subseteq J_{2} \subseteq J_{3} \subseteq \cdots$ be an ascending chain of $S$-perfect ideals in $R^{\prime}$ and let $J_{1}^{*} \subseteq J_{2}^{*} \subseteq J_{3}^{*} \subseteq \ldots$ be the chain of the corresponding perfect ideals in $R^{* \prime}$. Then for some $N \geqq 1, J_{N}^{*}=J_{N+1}^{*}=\cdots$ and hence $J_{N}=J_{N+1}=\cdots$.

As a corollary to 7.2 we have

7.3. If $R$ is a Ritt algebra then $R^{\prime}$ satisfies the finite ascending chain condition for $S$-perfect ideals.

Let $\widetilde{\Omega}=\left(\widetilde{R}, \widetilde{R}_{0}, \tilde{H}\right)$ be any 1.p.d.r. which is an extension of $\Omega$ and let $\widetilde{R}^{m}$ be the $m$-dimensional vector space over $\tilde{R}$. A vector $\eta=\left(\eta_{1}, \cdots, \eta_{m}\right)$ in $R^{m}$ is said to satisfy the polynomial $p\{u\}=p\left\{u_{1}, \cdots, u_{m}\right\} \in R^{\prime}$ if $p\left\{\eta_{1}, \cdots, \eta_{m}\right\}=0 . \eta$ is said to satisfy $q\left\{z_{10}, \cdots, z_{m 0}, z_{11}, \cdots, z_{m 1}, \cdots\right\} \in R_{0}$ if $\left.q\left\{H\left(\eta_{1}\right) \cdots H\left(\eta_{m}\right), H\left(\bar{D}_{n}\left(\eta_{1}\right)\right), \cdots, H\left(\bar{D}_{n}\left(\eta_{m}\right)\right), H\left(\bar{D}_{n}^{2}\left(\eta_{1}\right)\right), \cdots, H\left(\bar{D}_{n}^{2}\left(\eta_{m}\right)\right), \cdots\right)\right\}=0$.

We prove, as in [3], that the set of all polynomials in $R^{\prime}$ and $R_{0}^{\prime}$ which are satisfied by all the vectors of a given subset of $\widetilde{R}^{m}$ constitute a bi-ideal in $\Omega^{\prime}$. Given a bi-ideal $\alpha$ in $\Omega^{\prime}$ we again define the variety of $\alpha$ as the set of all vectors which satisfy the polynomials of $\alpha$, and we introduce the concept of a generic point, as before. 
7.4. TheOREM. Let $\alpha=\left(J, J_{0}\right)$ be a proper bi-ideal in $\Omega^{\prime}$. Then $\alpha$ possesses a generic point if and only if $\alpha$ is prime.

Proof. Suppose first that $\alpha$ is prime. Let $\widetilde{R}$ be the residue ring $R^{\prime} / J$. Since $J$ is prime, $\tilde{R}$ is a partial differential integral domain, and since $J$ is a proper $S$-ideal, it contains no elements of $R$ other than 0 . It follows that the images of the elements of $R$ in $R^{\prime} / J$ constitute a partial differential ring which is isomorphic to $R$. Thus, $\tilde{R}$ may be regarded as an extension of $R$, and similarly, $\widetilde{R}_{0}=R^{\prime} / J_{0}$ may be regarded as an extension of $R_{0}$. Also, the natural homomorphisms

$$
\Phi: R^{\prime} \rightarrow R^{\prime} / J \text { and } \Phi_{0}: R_{0}^{\prime} \rightarrow R_{0}^{\prime} / J_{0}
$$

are differential homomorphisms which map the elements of $R$ and $R_{0}$ (individually) on themselves.

Consider now the mapping $\Phi_{0} H^{\prime}$. This is a homomorphism from $R^{\prime}$ into $R_{0}$. Moreover, the kernel of $\Phi$ is contained in the kernel of $\Phi_{0} H^{\prime}$ so that the equation $\tilde{H} \Phi(a)=\Phi_{0} H(a)$, for $a \in R^{\prime}$, defines a unique homomorphism $\tilde{H}$ from $\widetilde{R}$ into $\widetilde{R}_{0}$. $\tilde{H}$ satisfies the condition (1.1) above since, for all $a \in R^{\prime}$,

$$
\begin{aligned}
\tilde{H}\left(\sum_{k=1}^{n} a_{i k} D_{k} \Phi(a)\right) & =\tilde{H} \Phi \sum_{k=1}^{n} a_{i k} D_{k} a=\Phi_{0} H^{\prime}\left(\sum_{k=1}^{n} a_{i k} D_{k} a\right) \\
& =\Phi_{0}\left(\Delta_{i} H^{\prime}(a)\right)=\Delta_{i} \Phi_{0} H^{\prime}(a)=\Delta_{i} \tilde{H} \Phi(a) .
\end{aligned}
$$

We have thus shown that $\tilde{\mathbf{\Omega}}=\left(\tilde{R}, \tilde{R}_{0}, \tilde{H}\right)$ is an 1.p.d.r. which is an extension $\Omega$. The required generic point is now given by $\eta=\left(\Phi\left(u_{1}\right), \cdots, \Phi\left(u_{m}\right)\right)$.

This proves the sufficiency of the condition of 7.4. Necessity is obvious.

7.5. THEOREM. Let $V=\bigcap_{\mu} V_{\mu}$ be the intersection of a set of varieties $\left\langle V_{\mu}\right\rangle$. Then $V$ is a variety.

Proof. For each $\mu$, let $V_{\mu}$ be the variety of a bi-ideal $\alpha_{\mu}=\left(J_{\mu}, J_{0 \mu}\right)$. Let $\alpha$ be the bi-ideal which is generated by the sets of polynomials $K=\bigcup_{\mu} J_{\mu}$ and $K_{0}=\bigcup_{\mu} J_{0 \mu}$, and let $V^{\prime}$ be the variety of $\alpha$. Then the elements of $V$ satisfy the polynomials of $K$ and $K_{0}$ and so $V \subseteq V^{\prime}$. On the other hand, any point which does not belong to $V$ will not satisfy some polynomials of $K$ or $K_{0}$ and hence can not belong to $V^{\prime}$. Hence $V=V^{\prime}, V$ is a variety.

As remarked in [3], the union of two varieties is not necessarily a variety.

The same methods as in [3] yield the relation $\alpha \rightarrow V \rightarrow \bar{\alpha}$ where $\bar{\alpha}$ is the closure of $\alpha$.

A system of polynomials $\left(K, K_{0}\right)$ in $\Omega^{\prime}$ is said to be consistent if the polynomials which belong to $K$ or $K_{0}$ possess a joint zero in some extension of $\Omega$. It follows from the preceding discussion that this will be the case if and only if the system $\left(K, K_{0}\right)$ generates a proper bi-ideal. Now by $(6.1)$, the bi-ideal generated by $\left(K, K_{0}\right)$ is $\left(J, J_{0}\right)=\left(\{K\}_{S},\left\{H^{\prime}\left(\{K\}_{1}\right) \cup K_{0}\right\}\right)$. Hence 
7.6. THEOREM. In order that the system $\left(K, K_{0}\right)$ be consistent, it is necessary and sufficient that

$$
1 \notin\left\{H^{\prime}\left(\{K\}_{S}\right) \cup K_{0}\right\} .
$$

For any set of polynomials $K$ (in $R^{\prime}$ or $R_{0}^{\prime}$ ), we denote by $d(K)$ the set of all derivatives of elements of $K$, including $K$. With this notation we may replace 7.6 by a more effective test in case $R_{0}$ is a Ritt algebra. Note that in that case $R$ also is a Ritt algebra. The test in question is then given by

7.7. TheOREM. Let $R$ be a Ritt algebra; then a system $\left(K, K_{0}\right)$ in $\Omega^{\prime}$ is consistent if and only if

$$
1 \notin\left(H^{\prime}\left([K]_{S}\right) \cup d\left(K_{0}\right)\right) .
$$

Proof. If $\left(J, J_{0}\right)$ is generated by $\left(K, K_{0}\right)$, then, in accordance with an earlier remark, a necessary and sufficient condition for consistency is $1 \notin J_{0}$. We shall show that if $R$ is a Ritt algebra, $J_{0}=\sqrt{ }\left(H^{\prime}\left([K]_{s}\right) \cup d\left(K_{0}\right)\right)$. But 1 belongs to the radical of an ideal if and only if it belongs to that ideal, and so the conclusion of the theorem will follow.

In order to establish the equation $J_{0}=\sqrt{ }\left(H^{\prime}\left([K]_{s}\right) \cup d\left(K_{0}\right)\right)$, we observe that

$$
J_{0}=\left\{H^{\prime}\left(\{K\}_{s}\right) \cup K_{0}\right\}=\sqrt{ }\left[H^{\prime}\left(\{K\}_{s}\right) \cup K_{0}\right]=\sqrt{ }\left(d\left(H^{\prime}\left(\{K\}_{s}\right)\right) \cup d\left(K_{0}\right)\right) .
$$

Now $H^{\prime}\left(\{K\}_{s}\right)$ is closed under differentiation, i.e., it is equal to $d\left(H^{\prime}\left(\{K\}_{s}\right)\right)$ for if $a$ is any element of $\{K\}_{s}$, then $D_{i}(a) \in\{K\}_{s}$ for all $D_{i}$ and so, for $i=1, \cdots, n$,

as required. Hence

$$
\Delta_{i} H^{\prime}(a)=H^{\prime}\left(\sum_{k=1}^{n} a_{t k} D_{k} a\right) \in H^{\prime}\left(\{K\}_{S}\right),
$$

$$
\begin{aligned}
J_{0} & =\sqrt{ }\left(d\left(H^{\prime}\left(\{K\}_{S}\right)\right) \cup d\left(K_{0}\right)\right)=\sqrt{ }\left(H^{\prime}\left(\sqrt{ }[K]_{S}\right) \cup d\left(K_{0}\right)\right) \\
& \subseteq \sqrt{ }\left(H^{\prime}\left([K]_{S}\right) \cup d\left(K_{0}\right)\right) \subseteq J_{0},
\end{aligned}
$$

and so

as required.

$$
J_{0}=\sqrt{ }\left(H^{\prime}\left([K]_{s}\right) \cup d\left(K_{0}\right)\right),
$$

Let $\left(K, K_{0}\right)$ be a system of polynomials as above and let $p\{u\}$ be any other polynomial in $R^{\prime}$. We may ask under what conditions $p\{u\}$ vanishes for all joint zeros of $\left(K, K_{0}\right)$. As in the case treated in [3], this will be the case if and only if $p$ belongs to all admissible prime components of $\{K\}_{s}$. (A prime component $J_{i}$ of $\{K\}_{S}$ is admissible if and only if the system $\left(J_{i}, K_{0}\right)$ is consistent.) In particular, we obtain the following result for Ritt algebras:

7.8. TheOREM. Let $\{K\}_{s}=J_{1} \cap \cdots \cap J_{k}$ be the representation of $\{K\}_{s}$ by its prime components. In order that the polynomial $p \in R^{\prime}$ vanish for all zeros 
of the system $\left(K, K_{0}\right)$, it is necessary and sufficient that for any $J_{i}(i=1, \cdots, k)$ either $p \in J_{i}$ or $1 \in\left(H^{\prime}\left(J_{i}\right) \cup d\left(K_{0}\right)\right)$, or both.

Again, we may ask under what conditions a polynomial $q \in R_{0}^{\prime}$ is satisfied by all zeros of $\left(K, K_{0}\right)$. For Ritt algebras, the answer to this question is provided by

7.9. THEOREM. In order that the polynomial $q\{z\} \in R_{0}^{\prime}$ vanish for all zeros of the system $\left(K, K_{0}\right)$, it is necessary and sufficient that there exists a positive integer $\rho$ such that

$$
q^{\rho} \in\left(H^{\prime}\left([K]_{S}\right) \cup d\left(K_{0}\right)\right) .
$$

Proof. Let $\alpha=\left(J, J_{0}\right)$ be the bi-ideal generated by $\left(K, K_{0}\right)$ and let $\bar{\alpha}=\left(\bar{J}_{,}, \bar{J}_{0}\right)$ be the closure of $\alpha$. Then $\bar{J}_{0}=J_{0}$ and $\alpha \rightarrow V \rightarrow \bar{\alpha}$ where $V$ is the variety of $\alpha$. Thus, $q$ vanishes for all zeros of $\left(K, K_{0}\right)$ if and only if $q \in \bar{J}_{0}=J_{0}$. But, as shown above (see the proof of 7.6), $J_{0}=\left(H^{\prime}\left(\sqrt{ }[K]_{s}\right) \cup d\left(K_{0}\right)\right)$. This proves 7.9.

Finally, we consider the classical Cauchy problem for a system of first order partial differential equations within the framework of local partial differential algebra.

7.10. THEOREM. Let $\Omega=\left(R, R_{0}, H\right)$ be an l.p.d.r. such that $R$ is a Ritt algebra. Let

$$
\vec{D}_{n} u_{i}=p_{i}\left(u_{1}, \cdots, u_{m}\right), \quad i=1, \cdots, m,
$$

be a set of partial differential equations with the "initial conditions",

$$
z_{i 0}=a_{i}, \quad i=1, \cdots, m,
$$

where $\bar{D}_{n}$ denotes the transversal derivative (the transversal vector having been specified in advance) and where

$$
p_{i} \in R\left(u_{1}, \cdots, u_{m}\right), \quad a_{i} \in R_{0}, \quad i=1, \cdots, m .
$$

Then (7.11), (7.12) possess a solution in some l.p.d.r. which is an extension of $\Omega$.

Proof. Let $K=\left\langle\bar{D}_{n} u_{i}-p_{i}\right\rangle, i=1, \cdots, m$, and $K_{0}=\left\langle z_{i o}-a_{i}\right\rangle, i=1, \cdots, m$. According to Theorem 7.7, the conclusion of 7.10 will have been proved if we can show that $1 \notin\left(H^{\prime}\left([K]_{s}\right) \cup d\left(K_{0}\right)\right)$. Suppose on the contrary that $1 \in\left(H^{\prime}\left([K]_{s} \cup d\left(K_{0}\right)\right)\right.$. If so, there exists an identity

$$
1=r_{1} t_{1}+\cdots+r_{k} t_{k}+s_{1} v_{1}+\cdots+s_{l} v_{l},
$$

where $r_{i}, s_{i} \in R_{0}^{\prime}, t_{i}=H\left(T_{i}\right)$, with $T_{i} \in[K]_{s}$ and $v_{i} \in d\left(K_{0}\right)$.

By 3.1, the $D_{i}$ are linear combinations of the $\bar{D}_{i}$. It follows, taking into account 7.1 , that we may write the $T_{i}$ in the form

$$
T_{\imath}=\sum_{j, \alpha_{1}, \ldots, \alpha_{n}} P_{j . \alpha_{1}, \ldots, \alpha_{n}} \bar{D}_{1}^{\alpha_{1}} \cdots \bar{D}_{n}^{\alpha_{n}}\left(\bar{D}_{n} u_{j}-P_{j}\right) \text {, }
$$

where the $P_{j, \alpha_{1}, \ldots, \alpha_{n}}$ belong to $R^{* \prime}$. 
Among all the terms which occur on the right hand side of the equations (7.14), we choose one for which $\alpha_{1}+\cdots+\alpha_{n}$ is as large as possible. Replacing $\bar{D}_{1}^{\alpha_{1}} \cdots \bar{D}_{n}^{\alpha_{n}+1} u_{j}$ in this term by $\bar{D}_{1}^{\alpha_{1}} \cdots \bar{D}_{n}^{\alpha_{n}} p_{j}$ eliminates the term without affecting the remaining expressions. Repeating this procedure a finite number of times we obtain 0 on all right hand sides of (7.14). Applying the corresponding substitutions in $R_{0}^{\prime}$, i.e., replacing expressions $\Delta_{1}^{\alpha_{1}} \cdots \Delta_{n-1}^{\alpha_{n-1}} z_{j, \alpha_{n}+1}=H^{\prime}\left(\bar{D}_{1}^{\alpha_{1}} \cdots \bar{D}_{n}^{\alpha_{n+1}} u_{j}\right)$ by $\Delta_{1}^{\alpha_{1}} \cdots \Delta_{n-1}^{\alpha_{n-1}}\left(H^{\prime} \bar{D}_{n}^{\alpha_{n}} p_{j}\right)$ in the same order, we eliminate $t_{1}, \cdots, t_{k}$ from (7.13), i.e., we obtain an identity of the form

$$
1=s_{1}^{\prime} v_{1}^{\prime}+\cdots+s_{1}^{\prime} v_{1}^{\prime},
$$

where $s_{i}^{\prime} \in R_{0}^{\prime}$ and $v_{i}^{\prime} \in d\left(K_{0}\right)$. But this identity expresses the fact that 1 belongs to the differential ideal $\left[K_{0}\right]$, and this is impossible since $\left[K_{0}\right]$ possesses the (differential) zero $z_{i o}=a_{i}, i=1, \cdots, m$. This shows that an identity of the type of (7.13) can not exist and completes the proof of the theorem.

The theory of regular local partial differential rings will be treated elsewhere.

\section{REFERENCES}

1. I. Kaplansky, An introduction to differential algebra, Actualités Sci. Indust. No. 1251, Hermann, Paris, 1957.

2. J. F. Ritt, Differential Algebra, Amer. Math. Soc. Colloq. Publ. Vol. 33, Amer. Math. Soc., Providence, R. I., 1950.

3. A. Robinson, Local differential algebra, Trans. Amer. Math. Soc. 97 (1960), 427-456.

HEBREW UNIVERSITY, JERUSALEM, ISRAEL 